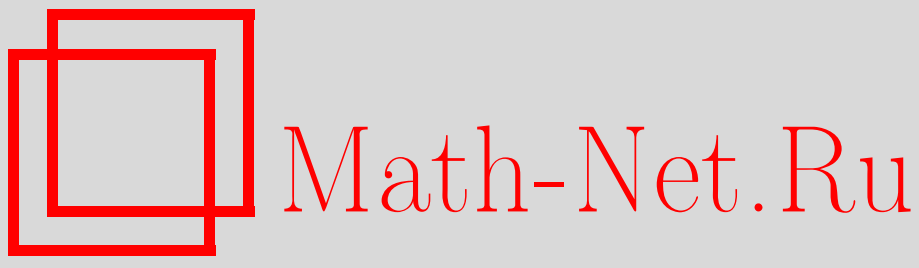

В. В. Корнев, А. П. Хромов, О равносходимости разложений по собственным функциям интегральных операторов с ядрами, допускающими разрывы производных на диагоналях, Матем. сб., 2001, том 192, номер 10, 33-50

DOI: https://doi.org/10.4213/sm601

Использование Общероссийского математического портала Math-Net.Ru подразумевает, что вы прочитали и согласны с пользовательским соглашением

http://www. mathnet.ru/rus/agreement

Параметры загрузки:

IP : 54.237 .59 .107

26 апреля 2023 г., 16:39:36 


\author{
В.В. Корнев, А.П. Хромов
}

\title{
О равносходимости разложений по собственным функциям интегральных операторов с ядрами, допускаюшими разрывы производных на диагоналях
}

В статьенайдены простыеусловия, при которых имеет место равносходимость разложений Фурье функции $f(x)$ из $L[0,1]$ по собственньм и присоединенньм функциям интегрального оператора вида

$$
A f=\int_{0}^{1-x} A(1-x, t) f(t) d t+\alpha \int_{0}^{x} A(x, t) f(t) d t
$$

и функций $f(x), f(1-x)$ по обычной тригонометрической системе.

Библиография: 4 названия.

\section{Введение}

В пространстве $L_{2}[0,1]$ рассмотрим интегральный оператор:

$$
A f=\int_{0}^{1-x} A(1-x, t) f(t) d t+\alpha \int_{0}^{x} A(x, t) f(t) d t, \quad x \in[0,1]
$$

где ядро $A(x, t) \quad n$ раз непрерьвно дифференцируемо по $x$ и один раз по $t$ при $0 \leqslant t \leqslant x \leqslant 1$ и

$$
\left.\frac{\partial^{j}}{\partial x^{j}} A(x, t)\right|_{t=x}=\delta_{n-1, j} \quad(j=0, \ldots, n),
$$

$\delta_{i, j}$ - символ Кронекера, $\alpha$ - произвольное число такое, что $\alpha^{2} \neq 1$.

Оператор (1) является одним из простейших операторов вида

$$
A f=\int_{0}^{1} A(x, t) f(t) d t
$$

некоторые производные ядер которых допускают разрывы первого рода на линиях $t=x$ и $t=1-x$. Другим важным достоинством оператора (1) является то, что в условиях получаемой теоремы равносходимости (в отличие от соответствующего результата из [1]) не требуется проверки труднопроверяемого условия регулярности по Биркгофу линейных форм в естественных граничных условиях. При $n=1$ и $\alpha=0$ равносходимость разложений по собственным и присоединенным функциям (с.п.ф.) и разложений в тригонометрические ряды Фурье установлена в [2]. Метод, примененный в нашей статье, является дальнейшим сушественным развитием

Работа вьполнена при финансовой поддержке Российского фонда фундаментальных исследований (грант № 00-01-00075) и Программы поддержки ведущих научных школ (грант № 00-15-96123). 
метода статьи [2]. Мы используем иное представление резольвенты простейшего оператора, и получение необходимых оценок компонент резольвенты является существенно более сложньм делом. Кроме того, пришлось отдельно рассматривать вопрос о равносходимости для случая простейшего оператора (в [2] этот момент является тривиальным), и еще следует отметить, что основное соотношение в теореме равносходимости имеет иной вид, не встречавшийся ранее. Есть основание ожидать, что требования гладкости на $A(x, t)$ ослабить нельзя, однако соответствующие контрпримеры (в отличие от [1]) построить не удалось.

\section{$\S 1$. Резольвента простейшего оператора}

Обозначим через $R_{\lambda}^{0}=\left(E-\lambda A_{0}\right)^{-1} A_{0}$ резольвенту Фредгольма простейшего оператора $A_{0}$ вида $(1)$, для которого $A(x, t)$ есть

$$
A_{0}(x, t)=\frac{(x-t)^{n-1}}{(n-1) !}
$$

( $E$ - единичный оператор, $\lambda$ - спектральный параметр). Для определенности будем считать, что $n$ четное (случай нечетного $n$ рассматривается аналогично). Рассмотрим следуюшую краевую задачу в пространстве двумерных вектор-функций:

$$
\begin{gathered}
z^{(n)}(x)-\lambda B_{1} z(x)=B_{1} F(x), \\
P_{1} z^{(j)}(0)+Q_{1} z^{(j)}(1)=0 \quad(j=0, \ldots, n-1),
\end{gathered}
$$

где

$$
B_{1}=\left(\begin{array}{cc}
\alpha & 1 \\
1 & \alpha
\end{array}\right), \quad P_{1}=\left(\begin{array}{cc}
0 & 0 \\
-\alpha & 1
\end{array}\right), \quad Q_{1}=\left(\begin{array}{cc}
1 & -\alpha \\
0 & 0
\end{array}\right)
$$

$z(x)=\left(z_{1}(x), z_{2}(x)\right)^{T}, F(x)=\left(F_{1}(x), F_{2}(x)\right)^{T}, F_{1}(x)=f(x), F_{2}(x)=f(1-x)$ ( $T$ - знак транспонирования).

Лемма 1. Если $\lambda$ таково, что $R_{\lambda}^{0}$ существует, то $z(x)$, где $z_{1}(x)=$ $R_{\lambda}^{0} f(x), z_{2}(x)=z_{1}(1-x)$, удовлетворяет (3)-(4). Обратно, если $z(x)$ удовлетворяет (3)-(4) и соответствующая однородная краевая задача имеет только нулевое решение, то $R_{\lambda}^{0}$ существует, $R_{\lambda}^{0} f(x)=z_{1}(x)$ u $z_{2}(x)=$ $z_{1}(1-x)$.

ДокАЗАТЕЛьство. Пусть $R_{\lambda}^{0}$ существует и $z_{1}(x)=R_{\lambda}^{0} f(x)$. Тогда имеем

$$
\begin{aligned}
z_{1}(x) & -\lambda\left(\int_{0}^{1-x} \frac{(1-x-t)^{n-1}}{(n-1) !} z_{1}(t) d t+\alpha \int_{0}^{x} \frac{(x-t)^{n-1}}{(n-1) !} z_{1}(t) d t\right) \\
= & \int_{0}^{1-x} \frac{(1-x-t)^{n-1}}{(n-1) !} f(t) d t+\alpha \int_{0}^{x} \frac{(x-t)^{n-1}}{(n-1) !} f(t) d t .
\end{aligned}
$$

Дифференцируя последовательно (5) до $n$-го порядка включительно, получаем

$$
\begin{gathered}
z_{1}^{(k)}(x)-\lambda\left((-1)^{k} \int_{0}^{1-x} \frac{(1-x-t)^{n-k-1}}{(n-k-1) !} z_{1}(t) d t+\alpha \int_{0}^{x} \frac{(x-t)^{n-k-1}}{(n-k-1) !} z_{1}(t) d t\right) \\
=(-1)^{k} \int_{0}^{1-x} \frac{(1-x-t)^{n-k-1}}{(n-k-1) !} f(t) d t+\alpha \int_{0}^{x} \frac{(x-t)^{n-k-1}}{(n-k-1) !} f(t) d t \\
z_{1}^{(k)}(1)=(-1)^{k} \alpha z_{1}^{(k)}(0) \quad(k=0, \ldots, n-1) \\
z_{1}^{(n)}(x)-\lambda\left(z_{1}(1-x)+\alpha z_{1}(x)\right)=f(1-x)+\alpha f(x)
\end{gathered}
$$


Если положить $z_{2}(x)=z_{1}(1-x)$, то из (6)-(8) следует (3)-(4).

Обратно, пусть $z(x)$ удовлетворяет (3)-(4) и соответствующая однородная задача имеет только нулевое решение. Тогда $u(x)=\left(u_{1}(x), u_{2}(x)\right)^{T}$, где $u_{1}(x)=$ $z_{2}(1-x)$ и $u_{2}(x)=z_{1}(1-x)$, также удовлетворяет (3)-(4). Поэтому $z(x)=u(x)$ и, значит, $z_{2}(x)=z_{1}(1-x)$. Покажем теперь, что $z_{1}(x)$ удовлетворяет $(5)$. Рассмотрим вспомогательную задачу:

$$
y^{\prime}(x)=g(x), \quad y(1)=\beta y(0) .
$$

Эта задача имеет и притом единственное решение при любой $g(x) \in L[0,1]$ тогда и только тогда, когда $\beta \neq 1$, и это решение дается формулой:

$$
y(x)=\int_{0}^{x} g(t) d t+\frac{1}{\beta-1} \int_{0}^{1} g(t) d t .
$$

Перепишем (8) в виде

$$
\left(z_{1}^{(n-1)}(x)\right)^{\prime}=\lambda\left(z_{1}(1-x)+\alpha z_{1}(x)\right)+f(1-x)+\alpha f(x)
$$

и добавим к нему последнее из соотношений (7):

$$
z_{1}^{(n-1)}(1)=-\alpha z_{1}^{(n-1)}(0)
$$

Рассматривая (11)-(12) как задачу (9), по формуле (10) после несложных преобразований приходим к (6) при $k=n-1$. Теперь, представляя (6) при $k=n-1$ и (7) при $k=n-2$ в виде (9), по формуле (10) приходим к (6) при $k=n-2$ и т. д. В конце концов получаем (5). Оператор $E-\lambda A_{0}$ обратим. В самом деле, если $v_{1}(x)-\lambda A_{0} v_{1}(x)=0$, то, как показано выше, $v(x)=\left(v_{1}(x), v_{2}(x)\right)^{T}$, где $v_{2}(x)=v_{1}(1-x)$, удовлетворяет (3)-(4) при $F(x)=0$. Значит, $v_{1}(x)=0$ и, тем самьм, $E-\lambda A_{0}$ обратим. Поэтому из (5) получаем $z_{1}(x)=R_{\lambda}^{0} f(x)$. Лемма доказана.

Таким образом, задача нахождения $R_{\lambda}^{0}$ сводится к решению краевой задачи (3)-(4). В свою очередь, используя подстановку:

$$
z(x)=\Gamma v(x), \quad \Gamma=\left(\begin{array}{cc}
1 & 1 \\
1 & -1
\end{array}\right)
$$

преобразуем задачу (3)-(4) к

$$
\begin{gathered}
v^{(n)}(x)-\lambda \mathscr{D} v(x)=B F(x) \\
P v^{(j)}(0)+Q v^{(j)}(1)=0 \quad(j=0, \ldots, n-1)
\end{gathered}
$$

где

$$
\begin{gathered}
\mathscr{D}=\left(\begin{array}{cc}
d_{1} & 0 \\
0 & d_{2}
\end{array}\right), \quad d_{1}=\alpha+1, \quad d_{2}=\alpha-1 \\
P=\left(\begin{array}{cc}
0 & 0 \\
p_{1} & p_{2}
\end{array}\right), \quad Q=\left(\begin{array}{cc}
p_{1} & -p_{2} \\
0 & 0
\end{array}\right), \quad p_{1}=1-\alpha, \quad p_{2}=-\alpha-1 \\
B=\frac{1}{2}\left(\begin{array}{cc}
\alpha+1 & \alpha+1 \\
\alpha-1 & 1-\alpha
\end{array}\right) .
\end{gathered}
$$


Займемся задачей (14)-(15). Обозначим через $V_{j}(x, \lambda)(j=1, \ldots, n)$ матрицы размера $2 \times 2$, которые образуют фундаментальную систему решений матричного уравнения $V^{(n)}-\lambda \mathscr{D} V=0$, а через $W(x, \lambda)$ их вронскиан, т.е. матрицу $W(x, \lambda)=\left(V_{j}^{(n-i)}(x, \lambda)\right)_{i, j=1}^{n}$ размера $2 n \times 2 n$. Матрица $W(x, \lambda)$ неособая, и, значит, $W^{-1}(x, \lambda)$ сушествует. Первые два столбца матрицы $W^{-1}(x, \lambda)$ разобьем сверху вниз на $n$ квадратных матриц $W_{j 1}(x, \lambda)(j=1, \ldots, n)$ и через $W_{j}(x, t, \lambda)$ $(j=1, \ldots, n)$ обозначим матрицы размера $2 \times 2$, элементы которых $\left(W_{j}(x, t, \lambda)\right)_{k l}$ $(k, l=1,2)$ определяются через соответствующие элементы матриц $W_{j 1}$ следуюшим образом:

$$
\left(W_{j}(x, t, \lambda)\right)_{k l}= \begin{cases}\left(W_{j 1}(t, \lambda)\right)_{k l}, & t \leqslant x \\ 0, & t>x\end{cases}
$$

или

$$
\left(W_{j}(x, t, \lambda)\right)_{k l}= \begin{cases}0, & t \leqslant x \\ -\left(W_{j 1}(t, \lambda)\right)_{k l}, & t>x .\end{cases}
$$

В дальнейшем будет указано правило выбора одной из этих формул для каждого $\left(W_{j}(x, t, \lambda)\right)_{k l}$.

Лемма 2. Общее решение системы (14) имеет вид:

$$
v(x, \lambda)=\sum_{j=1}^{n} V_{j}(x, \lambda) c_{j}+\int_{0}^{1} g(x, t, \lambda) B F(t) d t
$$

$2 \partial e$

$$
g(x, t, \lambda)=\sum_{j=1}^{n} V_{j}(x, \lambda) W_{j}(x, t, \lambda)
$$

и $c_{j}$ - произвольные постоянные векторы размерности 2.

ДокАЗАТЕЛЬство. Общее решение соответствуюшей однородной системы имеет вид:

$$
v(x, \lambda)=\sum_{j=1}^{n} V_{j}(x, \lambda) c_{j}
$$

Общее решение системы (14) ищем методом вариации произвольных постоянных и получаем следуюшую систему уравнений:

$$
\sum_{j=1}^{n} V_{j}^{(n-1)}(x, \lambda) c_{j}^{\prime}=B F(x), \quad \sum_{j=1}^{n} V_{j}^{(i)}(x, \lambda) c_{j}^{\prime}=0 \quad(i=0, \ldots, n-2) .
$$

Решениями системы (21) будут

$$
c_{j}^{\prime}=W_{j 1}(x, \lambda) B F(x) \quad(j=1, \ldots, n) .
$$

Интегрируя каждую скалярную компоненту системы (22) либо от 0 до $x$, либо от $x$ до 1 (как именно, об этом позже) и подставляя в (20), приходим к (18). Лемма доказана.

Введем матрицу $\Delta(\lambda)$ размера $2 n \times 2 n$ :

$$
\Delta(\lambda)=\left(U_{i j}(\lambda)\right)_{i, j=1}^{n},
$$

где $U_{i j}(\lambda)=U_{i}\left(V_{j}(x, \lambda)\right), U_{i}(V)=P V^{(i-1)}(0)+Q V^{(i-1)}(1)$. 
Теорема 1. Если $\lambda$ таково, что $\Delta^{-1}(\lambda)$ существует, то краевая задача (14)-(15) имеет единственное решение и это решение дается формулой:

$$
\begin{gathered}
v(x, \lambda)=-\left(V_{1}(x, \lambda), \ldots, V_{n}(x, \lambda)\right) \Delta^{-1}(\lambda) \int_{0}^{1} U_{x}(g(x, t, \lambda)) B F(t) d t \\
+\int_{0}^{1} g(x, t, \lambda) B F(t) d t
\end{gathered}
$$

әде $U_{x}(g)=\left(U_{1}^{T}(g), \ldots, U_{n}^{T}(g)\right)^{T}\left(U_{x}\right.$ означает, что $U_{j}$ применяются по переменной $x$ ).

ДокАЗАТЕЛЬСТво. Из (18) в силу $(16),(17)$ и (19) имеем

$$
v^{\prime}(x, \lambda)=\sum_{j=1}^{n} V_{j}^{\prime}(x, \lambda) c_{j}+\int_{0}^{1} g_{x}^{\prime}(x, t, \lambda) B F(t) d t+\sum_{j=1}^{n} V_{j}(x, \lambda) W_{j 1}(x, \lambda) B F(x)
$$

Последняя сумма в правой части представляет собой произведение двух последних строк матрицы $W(x, \lambda)$ и двух первых столбцов матрицы $W^{-1}(x, \lambda)$, и так как $W(x, \lambda) W^{-1}(x, \lambda)$ - единичная матрица, то эта сумма равна нулю. Аналогично обстоит дело и с другими производными $v^{(i)}(x, \lambda)(i=2, \ldots, n-1)$. Таким образом, получаем

$$
v^{(i)}(x, \lambda)=\sum_{j=1}^{n} V_{j}^{(i)}(x, \lambda) c_{j}+\int_{0}^{1} g_{x^{i}}(x, t, \lambda) B F(t) d t \quad(i=0, \ldots, n-1) .
$$

Подставляя теперь (25) в краевые условия (15), для нахождения $c_{j}$ получаем следующую систему линейных алгебраических уравнений:

$$
\Delta(\lambda) c+\int_{0}^{1} U_{x}(g(x, t, \lambda)) B F(t) d t=0
$$

где $c=\left(c_{1}^{T}, \ldots, c_{n}^{T}\right)^{T}$. Отсюда получим $c$ и, подставив его в $(18)$, придем к $(24)$. Теорема доказана.

СлЕДСТВИЕ. Если $\lambda$ таково, что $\Delta^{-1}(\lambda)$ существует, то $R_{\lambda}^{0}$ существует и имеет место формула:

$$
R_{\lambda}^{0} f(x)=v_{1}(x, \lambda)+v_{2}(x, \lambda)
$$

где $v(x, \lambda)=\left(v_{1}(x, \lambda), v_{2}(x, \lambda)\right)^{T}$ определяется по формуле $(24)$.

Это утверждение вытекает из доказанной теоремы, соотношения (13) и леммы 1. 


\section{§2. Оценки резольвенты простейшего оператора}

Займемся получением необходимых оценок $R_{\lambda}^{0}$ при больших значениях $|\lambda|$.

Для упрошения выкладок в (14) перейдем от параметра $\lambda$ к $\mu=\lambda(\alpha+1)$ (и $\mu$ снова обозначим через $\lambda$ ). Тогда в системе (14) матрица $\mathscr{D}$ станет такой $\left(\begin{array}{cc}1 & 0 \\ 0 & d_{2}\end{array}\right)$, где $d_{2}=(\alpha-1) /(\alpha+1)$.

Положим $\lambda=\rho^{n}(0 \leqslant \arg \rho \leqslant 2 \pi / n), \omega_{j}=\exp (2 \pi i(j-1) / n)(j=1, \ldots, n)$, $d=\left|d_{2}\right|^{1 / n} \exp \left(i \arg d_{2} / n\right)\left(0 \leqslant \arg d_{2}<2 \pi\right)$. Разобьем сектор $0 \leqslant \arg \rho \leqslant 2 \pi / n$ на секторы $\gamma_{s-1} \leqslant \arg \rho \leqslant \gamma_{s}\left(s=1, \ldots, m ; 0=\gamma_{0}<\gamma_{1}<\cdots<\gamma_{m}=\right.$ $2 \pi / n)$ таким образом, чтобы каждый сектор обладал следующим свойством: числа $\omega_{1}, \ldots, \omega_{n}, d \omega_{1}, \ldots, d \omega_{n}$ можно перенумеровать в таком порядке $\widetilde{\omega}_{1}, \widetilde{\omega}_{2}, \ldots, \widetilde{\omega}_{2 n}$, чтобы при любом $\rho$ из рассматриваемого сектора выполнялись неравенства:

$$
\operatorname{Re} \rho \widetilde{\omega}_{j} \geqslant 0 \quad(j=1, \ldots, n), \quad \operatorname{Re} \rho \widetilde{\omega}_{j} \leqslant 0 \quad(j=n+1, \ldots, 2 n),
$$

причем на одной из гранищ сектора $\operatorname{Re} \rho \widetilde{\omega}_{n}=\operatorname{Re} \rho \widetilde{\omega}_{n+1}=0$, а в случае $\arg d_{2}=0$ на этой же границе и $\operatorname{Re} \rho \widetilde{\omega}_{n-1}=\operatorname{Re} \rho \widetilde{\omega}_{n+2}=0$. Нетрудно видеть, что $m=4$ в случае $\arg d_{2}>0$ и $m=2$ в случае $\arg d_{2}=0$.

Для определенности рассмотрим сектор

$$
0 \leqslant \arg \rho \leqslant \gamma_{1}
$$

(другие секторы рассматриваются аналогично).

В качестве $V_{j}(x, \lambda)$ возьмем

$$
V_{j}(x, \lambda)=\left(\begin{array}{cc}
\exp \rho \omega_{j} x & 0 \\
0 & \exp \rho d \omega_{j} x
\end{array}\right) \quad(j=1, \ldots, n) .
$$

ЛЕмма 3. Для определителя матрицы $\Delta(\lambda)$ справедлива следующая асимптотическая формула:

$$
\begin{aligned}
& \operatorname{det} \Delta(\lambda)=\left(1-\alpha^{2}\right)^{n} \rho^{n(n-1)}\left[a_{0}+a_{1} \exp \left(-2 \rho \widetilde{\omega}_{n}\right)+a_{2} \exp \left(-\rho\left(\widetilde{\omega}_{n}+\widetilde{\omega}_{n-1}\right)\right)\right. \\
& \left.\quad+a_{3} \exp \left(-2 \rho \widetilde{\omega}_{n-1}\right)+a_{4} \exp \left(-2 \rho\left(\widetilde{\omega}_{n}+\widetilde{\omega}_{n-1}\right)\right)+o(1)\right] \exp \left(\rho \sum_{1}^{n} \widetilde{\omega}_{j}\right)
\end{aligned}
$$

причем $a_{0} \neq 0$.

ДокАЗАТЕЛЬСТво. В силу (23) и (29) нечетные строки $\Delta(\lambda)$ имеют вид:

$$
\begin{aligned}
p_{1}\left(\rho \omega_{1}\right)^{i-1} \exp \rho \omega_{1},- & p_{2}\left(\rho d \omega_{1}\right)^{i-1} \exp \rho d \omega_{1}, \ldots, \\
& p_{1}\left(\rho \omega_{n}\right)^{i-1} \exp \rho \omega_{n},-p_{2}\left(\rho d \omega_{n}\right)^{i-1} \exp \rho d \omega_{n},
\end{aligned}
$$

а четные:

$$
p_{1}\left(\rho \omega_{1}\right)^{i-1}, p_{2}\left(\rho d \omega_{1}\right)^{i-1}, \ldots, p_{1}\left(\rho \omega_{n}\right)^{i-1}, p_{2}\left(\rho d \omega_{n}\right)^{i-1} .
$$

Разлагая определитель этой матрицы по теореме Лапласа относительно строк с четными номерами, получаем, что

$$
\operatorname{det} \Delta(\lambda)=\left(1-\alpha^{2}\right)^{n} \rho^{n(n-1)} \sum_{j_{1}<\cdots<j_{n}} \gamma \exp \rho\left(\widetilde{\omega}_{j_{1}}+\cdots+\widetilde{\omega}_{j_{n}}\right),
$$


где через $\gamma$ обозначены постоянные числа, вообше говоря, различные. Из (31) и (27) в силу соотношений

$$
\widetilde{\omega}_{n}=-\widetilde{\omega}_{n+1}, \quad \widetilde{\omega}_{n-1}=-\widetilde{\omega}_{n+2}, \quad \exp \left(-\rho \widetilde{\omega}_{j}\right)=o(1) \quad(j=1, \ldots, n-2)
$$

следует представление (30). Далее, $a_{0}$ с точностью до знака равняется произведению определителей Вандермонда $n$-го порядка из наборов различных чисел $\widetilde{\omega}_{j}$. Лемма доказана.

ЛЕмМА 4. Удалим из сектора (28) все нули функиии $a_{0}+a_{1} \exp \left(-2 \rho \widetilde{\omega}_{n}\right)+$ $a_{2} \exp \left(-\rho\left(\widetilde{\omega}_{n}+\widetilde{\omega}_{n-1}\right)\right)+a_{3} \exp \left(-2 \rho \widetilde{\omega}_{n-1}\right)+a_{4} \exp \left(-2 \rho\left(\widetilde{\omega}_{n}+\widetilde{\omega}_{n-1}\right)\right)$ вместе $c$ круговыми окрестностями одного и того жсе достаточно малого радиуса $\delta_{0}$ и получившуюся область обозначим через $S_{\delta_{0}}$. Тогда в области $S_{\delta_{0}}$ при достаточно больиих $|\rho|$

$$
|\operatorname{det} \Delta(\lambda)| \geqslant c|\rho|^{n(n-1)}\left|\exp \left(\rho \sum_{j=1}^{n} \widetilde{\omega}_{j}\right)\right|,
$$

әде с >0 и не зависит от $\rho$.

ДокАЗАТЕЛьство. Если $\arg d_{2}>0$, то рассматриваемая функция имеет вид:

$$
a_{0}+a_{1} \exp \left(-2 \rho \widetilde{\omega}_{n}\right)+o(1)
$$

и утверждение $(32)$ легко следует из (30) (см. [3; $\S 4]$ ). Если $\arg d_{2}=0$, то $\widetilde{\omega}_{n-1}=$ $k \widetilde{\omega}_{n}(k-$ положительное число и $k \neq 1)$ и заменой $\tilde{\rho}=-\rho \widetilde{\omega}_{n}$ рассматриваемая функция приводится к квазимногочлену

$$
a_{0}+a_{1} \exp 2 \widetilde{\rho}+a_{2} \exp (k+1) \widetilde{\rho}+a_{3} \exp 2 k \widetilde{\rho}+a_{4} \exp 2(k+1) \widetilde{\rho},
$$

где $\operatorname{Im} \widetilde{\rho} \leqslant 0$ и $\widetilde{\rho}$ меняется в угловом секторе, прилегающем к мнимой оси. В этом случае утверждение (32) следует из представления (30) и оценки снизу модуля квазиполинома в области $S_{\delta_{0}}($ см. $[4 ; \S 12.5])$. Лемма доказана.

Зафиксируем одно из чисел $\widetilde{\omega}_{j}$ и обозначим

$$
\varphi(x, \rho ; f)=\left\{\begin{array}{l}
\int_{0}^{x} f(t) \exp \rho \widetilde{\omega}_{j}(x-t) d t, \quad \text { если } \operatorname{Re} \rho \widetilde{\omega}_{j} \leqslant 0 \\
\int_{x}^{1} f(t) \exp \rho \widetilde{\omega}_{j}(x-t) d t, \quad \text { если } \operatorname{Re} \rho \widetilde{\omega}_{j} \geqslant 0 .
\end{array}\right.
$$

Лемма 5. Справедливъц оценки:

$$
\begin{aligned}
& \|\varphi(x, \rho ; f)\|_{\infty}=O(1)\|f\|_{1}, \\
& \|\varphi(x, \rho ; f)\|_{\infty}=O\left(\varkappa\left(\left|\operatorname{Re} \rho \widetilde{\omega}_{j}\right|\right)\right)\|f\|_{\infty}, \\
& \|\varphi(x, \rho ; f)\|_{1}=O\left(\varkappa\left(\left|\operatorname{Re} \rho \widetilde{\omega}_{j}\right|\right)\right)\|f\|_{1}, \\
& \|\varphi(x, \rho ; \chi)\|_{\infty}=O\left(\frac{1}{|\rho|}\right)
\end{aligned}
$$

әде $\|\cdot\|_{\infty},\|\cdot\|_{1}$ - нормиц пространств $L_{\infty}[0,1]$ u $L[0,1], \chi(x)-$ характеристическая функиия отрезка $\left[\eta_{0}, \eta_{1}\right] \subset(0,1), \varkappa(y)=(1-\exp (-y)) / y$ при y $\geqslant 0$. Оценки $O(\cdot)$ равномерны по $\arg \rho$. 
ДокАЗАТЕльство. Оценка (34) вытекает из неположительности вещественной части показателя экспоненты в (33). Оценка (35) следует из того, что

$$
\begin{aligned}
& \int_{0}^{x}\left|\exp \rho \widetilde{\omega}_{j}(x-t)\right| d t=-\frac{1}{\operatorname{Re} \rho \widetilde{\omega}_{j}}\left(1-\exp \operatorname{Re} \rho \widetilde{\omega}_{j} x\right) \leqslant \varkappa\left(\left|\operatorname{Re} \rho \widetilde{\omega}_{j}\right|\right), \quad \operatorname{Re} \rho \widetilde{\omega}_{j} \leqslant 0 \\
& \int_{x}^{1}\left|\exp \rho \widetilde{\omega}_{j}(x-t)\right| d t=\frac{1-\exp \left(-\operatorname{Re} \rho \widetilde{\omega}_{j}(1-x)\right)}{\operatorname{Re} \rho \widetilde{\omega}_{j}} \leqslant \varkappa\left(\left|\operatorname{Re} \rho \widetilde{\omega}_{j}\right|\right), \quad \operatorname{Re} \rho \widetilde{\omega}_{j} \geqslant 0 .
\end{aligned}
$$

Докажем оценку (36). Для определенности рассмотрим верхнюю строку (33) (нижняя - аналогично). Имеем

$$
\begin{aligned}
\|\varphi(x, \rho ; f)\|_{1} & \leqslant \int_{0}^{1} d x \int_{0}^{x}|f(t)|\left|\exp \rho \widetilde{\omega}_{j}(x-t)\right| d t \\
& =\int_{0}^{1}|f(t)| \frac{1-\exp \left((1-t) \operatorname{Re} \rho \widetilde{\omega}_{j}\right)}{-\operatorname{Re} \rho \widetilde{\omega}_{j}} d t \leqslant \varkappa\left(\left|\operatorname{Re} \rho \widetilde{\omega}_{j}\right|\right)\|f\|_{1} .
\end{aligned}
$$

Наконец, при $f(t)=\chi(t)$ сначала вычисляем правую часть (33), а затем уже легко получаем (37). Лемма доказана.

ЗАмЕЧАниЕ. Утверждение леммы остается в силе, если $f(t)$ заменить на $f(1-t)$.

Лемма 6. Для әлементов $\eta_{i, j}(x, \lambda)(i=1,2 ; j=1, \ldots, 2 n)$ матрицы $\left(V_{1}(x, \lambda), \ldots, V_{n}(x, \lambda)\right) \Delta^{-1}(\lambda)$ в области $S_{\delta_{0}}$ справедливъ оценки:

$$
\begin{gathered}
\sum_{j=1}^{n}|\rho|^{j-1}\left(\left\|D^{m} \eta_{i, 2 j-1}(x, \lambda)\right\|_{\infty}+\left\|D^{m} \eta_{i, 2 j}(x, \lambda)\right\|_{\infty}\right)=O\left(|\rho|^{m}\right), \\
\sum_{j=1}^{n}|\rho|^{j-1}\left(\left\|D^{m} \eta_{i, 2 j-1}(x, \lambda)\right\|_{1}+\left\|D^{m} \eta_{i, 2 j}(x, \lambda)\right\|_{1}\right)=O\left(|\rho|^{m} \psi(\rho)\right) \\
(i=1,2 ; \quad j=0,1, \ldots, n-1),
\end{gathered}
$$

где $\psi(\rho)=\sum_{j=1}^{n} \varkappa\left(\operatorname{Re} \rho \widetilde{\omega}_{j}\right), D=d / d x$.

ДокаЗАТЕЛьство. В силу (29) имеем

$$
\begin{aligned}
& \eta_{1, j}(x, \lambda)=\sum_{k=1}^{n} A_{j, 2 k-1}(\rho)(\operatorname{det} \Delta(\lambda))^{-1} \exp \rho \widetilde{\omega}_{2 k-1} x, \\
& \eta_{2, j}(x, \lambda)=\sum_{k=1}^{n} A_{j, 2 k}(\rho)(\operatorname{det} \Delta(\lambda))^{-1} \exp \rho \widetilde{\omega}_{2 k} x
\end{aligned}
$$

где $A_{j, k}(\rho)$ - алгебраическое дополнение элемента с номером $(j, k)$ матрицы $\Delta(\lambda)$.

Рассмотрим $\eta_{2,2 l}(x, \lambda)$ (остальные функции рассматриваются аналогично). Запишем каждое слагаемое во второй формуле (40) в виде:

$$
\begin{array}{ll}
{\left[A_{2 l, 2 k}(\rho)(\operatorname{det} \Delta(\lambda))^{-1} \exp \rho \widetilde{\omega}_{2 k}\right] \exp \rho \widetilde{\omega}_{2 k}(x-1),} & \text { если } \operatorname{Re} \rho \widetilde{\omega}_{2 k} \geqslant 0, \\
{\left[A_{2 l, 2 k}(\rho)(\operatorname{det} \Delta(\lambda))^{-1}\right] \exp \rho \widetilde{\omega}_{2 k} x,} & \text { если } \operatorname{Re} \rho \widetilde{\omega}_{2 k} \leqslant 0 .
\end{array}
$$


Из структуры $\Delta(\lambda)$ следует, что алгебраическое дополнение $A_{2 l, 2 k}(\rho)$ не содержит $\exp \rho \widetilde{\omega}_{2 k}$. Поэтому по лемме 4 каждая из квадратных скобок имеет оценку $O\left(\rho^{1-l}\right)$ и, значит,

$$
D^{m} \eta_{2,2 l}(x, \lambda)=O\left(\rho^{1-l+m}\right) \quad(l=1, \ldots, n ; m=0,1, \ldots, n-1) .
$$

Аналогично оценка (41) получается для $\eta_{1,2 l}, \eta_{1,2 l-1}$ и $\eta_{2,2 l-1}$. Тем самым получена оценка (38) при $m=0$. Что касается остальных оценок (38) и (39), то они следуют из вьшеприведенного рассуждения и равенств:

$$
\begin{array}{ll}
\int_{0}^{1}\left|\exp \rho \widetilde{\omega}_{k}(x-1)\right| d x=\varkappa\left(\operatorname{Re} \rho \widetilde{\omega}_{k}\right), & \operatorname{Re} \rho \widetilde{\omega}_{k} \geqslant 0, \\
\int_{0}^{1}\left|\exp \rho \widetilde{\omega}_{k} x\right| d x=\varkappa\left(-\operatorname{Re} \rho \widetilde{\omega}_{k}\right), & \operatorname{Re} \rho \widetilde{\omega}_{k} \leqslant 0 .
\end{array}
$$

Лемма доказана.

В дальнейшем определимся с выбором матрищы $g(x, t, \lambda)$. Именно будем считать, что

$$
g(x, t, \lambda)=\frac{1}{n \rho^{n-1}}\left(\begin{array}{c}
\sum_{j=1}^{n} \omega_{j}\left(\gamma_{1, j} \varepsilon(x, t)+\gamma_{2, j} \varepsilon(t, x)\right) \exp \rho \omega_{j}(x-t) ; \quad 0 \\
0 ; \quad \frac{d}{d_{2}} \sum_{j=1}^{n} \omega_{j}\left(\widetilde{\gamma}_{1, j} \varepsilon(x, t)+\widetilde{\gamma}_{2, j} \varepsilon(t, x)\right) \exp \rho d \omega_{j}(x-t)
\end{array}\right),
$$

где $\varepsilon(x, t)=1$ при $t \leqslant x, \varepsilon(x, t)=0$ при $t>x ; \gamma_{1, j}=1, \gamma_{2, j}=0$, если $\operatorname{Re} \rho \omega_{j} \leqslant 0$; $\gamma_{1, j}=0, \gamma_{2, j}=-1$, если $\operatorname{Re} \rho \omega_{j} \geqslant 0 ; \widetilde{\gamma}_{1, j}=1, \widetilde{\gamma}_{2, j}=0$, если $\operatorname{Re} \rho d \omega_{j} \leqslant 0 ; \widetilde{\gamma}_{1, j}=0$, $\widetilde{\gamma}_{2, j}=-1$, если $\operatorname{Re} \rho d \omega_{j} \geqslant 0$.

Убедимся, что такой выбор $g(x, t, \lambda)$ возможен. Рассуждаем следуюшим образом. Матришы $W_{j 1}(x, \lambda)$ определяются из системы:

$$
\sum_{j=1}^{n} V_{j}^{(i)}(x, \lambda) W_{j 1}(x, \lambda)=\delta_{n-1, i} E \quad(i=0, \ldots, n-1)
$$

( $E$ - единичная матрица). В то же время непосредственно легко видеть, что матрицы

$$
\frac{\omega_{j}}{n \rho^{n-1}}\left(\begin{array}{cc}
\exp \left(-\rho \omega_{j} x\right) & 0 \\
0 & \frac{d}{d_{2}} \exp \left(-\rho d \omega_{j} x\right)
\end{array}\right),
$$

если их брать в качестве $W_{j 1}(x, \lambda)$, удовлетворяют (43). Поэтому $W_{j 1}(x, \lambda)$ равны (44) и матрицы $V_{j}(x, \lambda) W_{j}(x, t, \lambda)$ также диагональны, причем

$$
\left(V_{j}(x, \lambda) W_{j}(x, t, \lambda)\right)_{1,1}=\frac{\omega_{j}}{n \rho^{n-1}}\left(\gamma_{1} \varepsilon(x, t)+\gamma_{2} \varepsilon(t, x)\right) \exp \rho \omega_{j}(x-t),
$$

где $\gamma_{1}=1, \gamma_{2}=0$, если используется (16); $\gamma_{1}=0, \gamma_{2}=-1$, если используется (17). Для $\left(V_{j}(x, \lambda) W_{j}(x, t, \lambda)\right)_{2,2}$ справедлива формула (45) с добавлением множителя $d / d_{2}$ и множителя $d$ в показателе экспоненты. Теперь мы будем считать, что в случае $\operatorname{Re} \rho \widetilde{\omega}_{j} \leqslant 0$ используется (16), а в случае $\operatorname{Re} \rho \widetilde{\omega}_{j} \geqslant 0$ используется (17), и тем самым приходим к (42).

Представление (42) замечательно тем, что матрица $n \rho^{n-1} g(x, t, \lambda)$ при $t \leqslant x$ и $t \geqslant x$ представляет собой линейные комбинации $\exp \rho \widetilde{\omega}_{j}(x-t)$ с неположительными вешественными частями. 
ТЕОРема 2. В области $S_{\delta_{0}}$ при больиих $|\rho|$ справедливь оценки:

$$
\begin{gathered}
\left\|R_{\lambda}^{0} f\right\|_{\infty}=O\left(|\rho|^{1-n}\right)\|f\|_{1}, \\
\left\|R_{\lambda}^{0} f\right\|_{\infty}=O\left(|\rho|^{1-n} \psi(\rho)\right)\|f\|_{\infty}, \\
\left\|D^{m} R_{\lambda}^{0} f\right\|_{1}=O\left(|\rho|^{1-n+m} \psi(\rho)\right)\|f\|_{1} \quad(m=0, \ldots, n-1), \\
\left\|D^{m} R_{\lambda}^{0} \chi\right\|_{\infty}=O\left(|\rho|^{-n+m}\right) \quad(m=0, \ldots, n-1),
\end{gathered}
$$

әде $D, \chi(x)$ и $\psi(\rho)$ те же, что и в леммах 5 и 6.

ДокАЗАТЕЛЬство. При доказательстве теоремы 1 было установлено, что

$$
D^{m} \int_{0}^{1} g(x, t, \lambda) B F(t) d t=\int_{0}^{1} D^{m} g(x, \rho ; f) B F(t) d t \quad(m=0, \ldots, n-1) .
$$

Поэтому в силу (42) имеем

$$
D^{m} \int_{0}^{1} g(x, t, \lambda) B F(t) d t=\rho^{1-n+m}\left(g_{1}(x, \rho ; f), g_{2}(x, \rho ; f)\right)^{T}
$$

где $g_{i}(x, \rho ; f)$ представляют собой линейные комбинации функций вида $(33)$ с не зависящими от $\rho$ коэффициентами, причем в (33) берутся как $f(t)$, так и $f(1-t)$. Тогда по лемме 5 и замечанию к ней получаем оценки:

$$
\begin{aligned}
& \left\|g_{i}(x, \rho ; f)\right\|_{\infty}=O(1)\|f\|_{1} \\
& \left\|g_{i}(x, \rho ; f)\right\|_{\infty}=O(\psi(\rho))\|f\|_{\infty}, \\
& \left\|g_{i}(x, \rho ; f)\right\|_{1}=O(\psi(\rho))\|f\|_{1} \\
& \left\|g_{i}(x, \rho ; \chi)\right\|_{\infty}=O\left(\frac{1}{|\rho|}\right) \quad(i=1,2) .
\end{aligned}
$$

Из определения матрицы $U_{x}(g)$ в силу (42) получаем представление:

$$
\begin{gathered}
\int_{0}^{1} U_{x}(g(x, t, \lambda)) B F(t) d t=\left(\rho^{1-n} \psi_{1}(\rho ; f), \rho^{1-n} \psi_{2}(\rho ; f),\right. \\
\left.\rho^{2-n} \psi_{3}(\rho ; f), \rho^{2-n} \psi_{4}(\rho ; f), \ldots, \psi_{2 n-1}(\rho ; f), \psi_{2 n}(\rho ; f)\right)^{T}
\end{gathered}
$$

где каждая из $\psi_{j}(\rho ; f)$ есть линейная комбинация с не зависящими от $\rho$ коэффициентами функций вида (33) в точках $x=0$ и $x=1$, причем в (33) берутся как $f(t)$, так и $f(1-t)$. Поэтому по лемме 5 и замечанию к ней имеем оценки:

$$
\begin{aligned}
& \psi_{j}(\rho ; f)=O(1)\|f\|_{1} \\
& \psi_{j}(\rho ; f)=O(\psi(\rho))\|f\|_{\infty} \\
& \psi_{j}(\rho ; \chi)=O\left(\frac{1}{|\rho|}\right) \quad(j=1, \ldots, 2 n) .
\end{aligned}
$$


Далее, в силу (24), (26), (50) и (55) имеем

$$
\begin{aligned}
D^{m} R_{\lambda}^{0} f(x)=\sum_{i=1}^{2} & {\left[\rho^{1-n+m} g_{i}(x, \rho ; f)\right.} \\
& \quad-\rho^{1-n} \sum_{j=1}^{n} \rho^{1-j}\left(D^{m} \eta_{i, 2 j-1}(x, \lambda) \psi_{2 j-1}(\rho ; f)\right. \\
& \left.\left.+D^{m} \eta_{i, 2 j}(x, \lambda) \psi_{2 j}(\rho ; f)\right)\right] \quad(m=0, \ldots, n-1) .
\end{aligned}
$$

При $m=0$ из этого представления и оценок $(51),(56)$ и (38) вытекает оценка (46), а из (52), (57) и (38) следует (47). Для получения (48) надо воспользоваться оценками (53), (56) и (39). Наконец, оценки (49) есть следствия представления (59), оценок $(54),(58)$ и (38). Теорема доказана.

\section{§ 3. Равносходимость спектральных разложений исходного и простейшего операторов}

Перейдем к изучению оператора (1). Введем в рассмотрение операторы:

$$
T_{1} f(x)=\int_{0}^{x} \frac{\partial^{n}}{\partial x^{n}} A(x, t) f(t) d t, \quad T=\left(E+T_{1}\right)^{-1}-E
$$

Из соотношения для ядер $T_{1}(x, t)$ и $T(x, t)$ операторов $T_{1}$ и $T$

$$
T_{1}(x, t)+T(x, t)+\int_{t}^{x} T(x, \tau) T_{1}(\tau, t) d \tau=0
$$

и $(2)$ следует, что $T(x, t)$ непрерывно дифференцируемо по $t$ и $T(x, x)=0$. Введем еще операторы:

$$
T_{t}^{\prime} f(x)=\int_{0}^{x} T_{t}^{\prime}(x, t) f(t) d t, \quad D=\frac{d}{d x}, \quad S f(x)=f(1-x)
$$

Лемма 7. Для резольвенты $R_{\lambda}=(E-\lambda A)^{-1}$ A имеет место формула

$$
R_{\lambda}=R_{\lambda}^{0}+\frac{1}{1-\alpha^{2}} R_{\lambda}^{0} T_{t}^{\prime} D^{n-1}(S-\alpha E) R_{\lambda}
$$

ДокАЗАТЕЛЬСТво. Положим $y(x)=R_{\lambda} f(x)$. Тогда

$$
\begin{gathered}
y(x)-\lambda\left[\int_{0}^{1-x} A(1-x, t) y(t) d t+\alpha \int_{0}^{x} A(x, t) y(t) d t\right] \\
=\int_{0}^{1-x} A(1-x, t) f(t) d t+\alpha \int_{0}^{x} A(x, t) f(t) d t .
\end{gathered}
$$


Дифференцируя (61) $n$ раз с учетом (2), получаем ${ }^{1}$

$$
\begin{aligned}
& D^{i} y(x)-\lambda\left[(-1)^{i} \int_{0}^{1-x} A_{x^{i}}(1-x, t) y(t) d t+\alpha \int_{0}^{x} A_{x^{i}}(x, t) y(t) d t\right] \\
& \quad=(-1)^{i} \int_{0}^{1-x} A_{x^{i}}(1-x, t) f(t) d t+\alpha \int_{0}^{x} A_{x^{i}}(x, t) f(t) d t \quad(i=0, \ldots, n-1) ; \\
& D^{n} y(x)-\lambda\left[y(1-x)+\int_{0}^{1-x} A_{x^{n}}(1-x, t) y(t) d t+\alpha y(x)+\alpha \int_{0}^{x} A_{x^{n}}(x, t) y(t) d t\right] \\
& \quad=f(1-x)+\int_{0}^{1-x} A_{x^{n}}(1-x, t) f(t) d t+\alpha f(x)+\alpha \int_{0}^{x} A_{x^{n}}(x, t) f(t) d t .
\end{aligned}
$$

Из соотношений (62) следует, что

$$
y^{(i)}(1)=(-1)^{i} \alpha y^{(i)}(0) \quad(i=0, \ldots, n-1) .
$$

Перепишем (63) в виде:

$$
D^{n} y(x)-\lambda(S+\alpha E)\left(E+T_{1}\right) y(x)=(S+\alpha E)\left(E+T_{1}\right) f(x) .
$$

Применяя к обеим частям (65) оператор $S$ и учитывая, что $n$ четное, получаем

$$
D^{n} S y(x)-\lambda(E+\alpha S)\left(E+T_{1}\right) y(x)=(E+\alpha S)\left(E+T_{1}\right) f(x) .
$$

Из (65) и (66) следует, что

$$
\frac{1}{1-\alpha^{2}} D^{n}(S-\alpha E) y(x)-\lambda\left(E+T_{1}\right) y(x)=\left(E+T_{1}\right) f(x) .
$$

В свою очередь, из (67) получаем

$$
\frac{1}{1-\alpha^{2}} D^{n}(S-\alpha E) y(x)-\lambda y(x)=f(x)-\frac{1}{1-\alpha^{2}} T D^{n}(S-\alpha E) y(x) .
$$

Отсюда на основании (64) и формулы $A_{0}^{-1}=\left(1-\alpha^{2}\right)^{-1} D^{n}(S-\alpha E)$ заключаем, что

$$
y(x)=R_{\lambda}^{0} f(x)-\frac{1}{1-\alpha^{2}} R_{\lambda}^{0} T D^{n}(S-\alpha E) y(x) .
$$

Так как $T D^{n}(S-\alpha E)=-T_{t}^{\prime} D^{n-1}(S-\alpha E)$, то из (68) вытекает (60). Лемма доказана.

Лемма 8. В области $S_{\delta_{0}}$ ядро интегрального оператора $D^{n-1}(S-\alpha E) R_{\lambda}^{0} T_{t}^{\prime}$ есть о(1) при $|\rho| \rightarrow \infty$ равномерно по остальным переменным.

$$
{ }^{1} \text { Считаем, что } A_{x^{i}}(1-x, t)=\left.\frac{\partial^{i}}{\partial \xi^{i}} A(\xi, t)\right|_{\xi=1-x}
$$


ДокАЗАТЕЛьство. Очевидно, утверждение леммы достаточно доказать для оператора $D^{n-1} R_{\lambda}^{0} T_{t}^{\prime}$. По формуле (59) при $m=n-1$ имеем

$$
\begin{aligned}
D^{n-1} R_{\lambda}^{0} T_{t}^{\prime} f(x)= & \sum_{i=1}^{2}\left[g_{i}\left(x, \rho ; T_{t}^{\prime} f\right)\right. \\
& -\rho^{1-n} \sum_{j=1}^{n} \rho^{1-j}\left(D^{n-1} \eta_{i, 2 j-1}(x, \lambda) \psi_{2 j-1}\left(\rho ; T_{t}^{\prime} f\right)\right. \\
& \left.\left.+D^{n-1} \eta_{i, 2 j}(x, \lambda) \psi_{2 j}\left(\rho ; T_{t}^{\prime} f\right)\right)\right] .
\end{aligned}
$$

Подставляя в (33) вместо $T_{t}^{\prime} f$ ее явное выражение и меняя порядок интегрирования, имеем

$$
\varphi\left(x, \rho ; T_{t}^{\prime} f\right)= \begin{cases}\int_{0}^{x} f(\tau) d \tau \int_{\tau}^{x} T_{\tau}^{\prime}(t, \tau) \exp \rho \widetilde{\omega}_{j}(x-t) d t, & \operatorname{Re} \rho \widetilde{\omega}_{j} \leqslant 0, \\ \int_{0}^{x} f(\tau) d \tau \int_{x}^{1} T_{\tau}^{\prime}(t, \tau) \exp \rho \widetilde{\omega}_{j}(x-t) d t & \\ +\int_{x}^{1} f(\tau) d \tau \int_{\tau}^{1} T_{\tau}^{\prime}(t, \tau) \exp \rho \widetilde{\omega}_{j}(x-t) d t, & \operatorname{Re} \rho \widetilde{\omega}_{j} \geqslant 0 .\end{cases}
$$

Применяя лемму 4 из [1] к внутренним интегралам в (70), заключаем, что

$$
\varphi\left(x, \rho ; T_{t}^{\prime} f\right)=\int_{0}^{1} o(1) f(t) d t
$$

Поэтому такое же представление имеют $g_{i}\left(x, \rho ; T_{t}^{\prime} f\right)(i=1,2)$ и $\psi_{j}\left(\rho ; T_{t}^{\prime} f\right)$ $(j=1, \ldots, n)$, и утверждение леммы следует из $(69)$ и $(38)$.

Лемма 9. В области $S_{\delta_{0}}$ справедливъ оценки:

$$
\begin{aligned}
& \left\|\left(R_{\lambda}-R_{\lambda}^{0}\right) f\right\|_{\infty}=O\left(|\rho|^{1-n} \psi^{2}(\rho)\right)\|f\|_{1}, \\
& \left\|\left(R_{\lambda}-R_{\lambda}^{0}\right) \chi\right\|_{\infty}=O\left(|\rho|^{-n} \psi(\rho)\right),
\end{aligned}
$$

әде $\chi(x)$ и $\psi(\rho)$ те жее, что и в леммах 5 и 6.

ДоказАтЕльство. По лемме 8 оператор $E-\left(1-\alpha^{2}\right)^{-1} D^{n-1}(S-\alpha E) R_{\lambda}^{0} T_{t}^{\prime}$ при достаточно больших $|\rho|$ обратим и этот обратный ограничен по $|\rho|$. Поэтому из (60) получаем

$$
R_{\lambda}=R_{\lambda}^{0}+\frac{1}{1-\alpha^{2}} R_{\lambda}^{0} T_{t}^{\prime}\left(E-\frac{1}{1-\alpha^{2}} D^{n-1}(S-\alpha E) R_{\lambda}^{0} T_{t}^{\prime}\right)^{-1} D^{n-1}(S-\alpha E) R_{\lambda}^{0} .
$$

Отсюда в силу (47) и (48) при $m=n-1$ имеем

$$
\begin{aligned}
& \left\|\left(R_{\lambda}-R_{\lambda}^{0}\right) f\right\|_{\infty}=O\left(|\rho|^{1-n} \psi(\rho)\right) \\
& \quad \times\left\|T_{t}^{\prime}\left(E-\frac{1}{1-\alpha^{2}} D^{n-1}(S-\alpha E) R_{\lambda}^{0} T_{t}^{\prime}\right)^{-1} D^{n-1}(S-\alpha E) R_{\lambda}^{0} f\right\|_{\infty} \\
& =O\left(|\rho|^{1-n} \psi(\rho)\right)\left\|D^{n-1}(S-\alpha E) R_{\lambda}^{0} f\right\|_{1}=O\left(|\rho|^{1-n} \psi^{2}(\rho)\right)\|f\|_{1},
\end{aligned}
$$

что доказывает (71). Для доказательства (72) вместо (48) надо воспользоваться (49) при $m=n-1$. Лемма доказана.

Все предыдущие оценки получены в случае (28). В остальных секторах $\gamma_{s-1} \leqslant$ $\arg \rho \leqslant \gamma_{s}$ аналогичньми рассуждениями эти оценки получаются в соответствующих областях $S_{\delta_{0}}$. Теперь, не меняя обозначений, будем считать, что $S_{\delta_{0}}$ есть объединение всех этих областей. 
ТеОрема 3. Для любой $f(x) \in L[0,1]$ имеет место

$$
\lim _{r \rightarrow \infty}\left\|\Omega_{r} f\right\|_{\infty}=0
$$

əдe

$$
\Omega_{r} f=\frac{1}{2 \pi i} \int_{|\lambda|=r}\left(R_{\lambda}-R_{\lambda}^{0}\right) f(x) d \lambda
$$

ur таково, ито $\left\{\rho:|\rho|=r^{1 / n}, 0 \leqslant \arg \rho \leqslant 2 \pi / n\right\} \subset S_{\delta_{0}}$.

ДокаЗАтЕльство. В (73) выполним замену: $\lambda=\rho^{n}, \rho=|\rho| \exp i \varphi, 0 \leqslant \varphi \leqslant$ $2 \pi / n$. Тогда имеем

$$
\Omega_{r} f=\frac{n}{2 \pi} \int_{0}^{2 \pi / n} \rho^{n}\left(R_{\lambda}-R_{\lambda}^{0}\right) f d \varphi=\frac{n}{2 \pi}\left(\int_{0}^{\gamma_{1}}+\int_{\gamma_{1}}^{\gamma_{2}}+\int_{\gamma_{2}}^{\gamma_{3}}+\int_{\gamma_{3}}^{2 \pi / n}\right) .
$$

Рассмотрим первый интеграл в (74). По лемме 9 имеем

$$
\int_{0}^{\gamma_{1}}=O\left(|\rho| \int_{0}^{\gamma_{1}} \psi^{2}(\rho) d \varphi\right)\|f\|_{1}
$$

Покажем, что

$$
|\rho| \int_{0}^{\gamma_{1}} \psi^{2}(\rho) d \varphi=O(1) .
$$

Предположим, что $\arg d_{2}>0$ (случай $\arg d_{2}=0$ рассматривается аналогично). Очевидно, что

$$
\varkappa\left(\operatorname{Re} \rho \widetilde{\omega}_{j}\right)=O\left(\frac{1}{|\rho|}\right) \quad(j=1, \ldots, n-1), \quad \varkappa\left(\operatorname{Re} \rho \widetilde{\omega}_{n}\right)=O(1) .
$$

Поэтому

$$
|\rho| \int_{0}^{\gamma_{1}} \psi^{2}(\rho) d \varphi=O(1)+|\rho| \int_{0}^{\gamma_{1}} \varkappa^{2}\left(\operatorname{Re} \rho \widetilde{\omega}_{n}\right) d \varphi
$$

Для последнего слагаемого в правой части (76) имеем оценку:

$$
\begin{aligned}
|\rho| \int_{0}^{\gamma_{1}} & =|\rho| \int_{0}^{\gamma_{1}}\left(\frac{1-\exp \left(-\left|\rho \widetilde{\omega}_{n}\right| \sin \varphi\right)}{\left|\rho \widetilde{\omega}_{n}\right| \sin \varphi}\right)^{2} d \varphi \\
& \leqslant \frac{\pi}{2\left|\widetilde{\omega}_{n}\right|} \int_{0}^{\infty}\left(\frac{1-\exp (-x)}{x}\right)^{2} d x<\infty
\end{aligned}
$$

и тем самьм оценка (75) установлена. Аналогично оцениваются и остальные интегралы в (74). Значит,

$$
\left\|\Omega_{r} f\right\|_{\infty}=O(1)\|f\|_{1} .
$$

Пусть теперь в $(74) f(x)=\chi(x)$. Тогда по лемме 9 первый интеграл в (74) имеет оценку

$$
\int_{0}^{\gamma_{1}}=O\left(\int_{0}^{\gamma_{1}} \psi(\rho) d \varphi\right) .
$$

Из (78) и (75), используя неравенство Коши-Буняковского, получаем, что $\int_{0}^{\gamma_{1}}=O\left(|\rho|^{-1 / 2}\right)$. Эта оценка аналогично устанавливается и для других интегралов в (74). Таким образом, утверждение теоремы получено для $f(x)=\chi(x)$. А тогда в силу (77) и теоремы Банаха-Штейнгауза оно имеет место и для произвольной функции $f(x) \in L[0,1]$. 
ЗАмЕчАниЕ. Так как $\Omega_{r} f$ равняется разности частных сумм разложений по собственным и присоединенным функциям (с.п.ф.) операторов $A_{0}$ и $A$ для тех характеристических значений, которые попадают в круг $|\lambda|<r$, то теорема 3 дает нам равносходимость спектральных разложений для $A$ и $A_{0}$.

\section{§4. Основная теорема}

Приступаем, наконец, к вопросу о равносходимости разложений по с.п.ф. оператора $A$ и разложений в тригонометрические ряды Фурье.

Введем следуюшую краевую задачу:

$$
\begin{gathered}
u^{(n)}-\lambda \mathscr{D} u=B F(x), \\
u^{(i)}(0)=u^{(i)}(1) \quad(i=0, \ldots, n-1),
\end{gathered}
$$

где $u(x)=\left(u_{1}(x), u_{2}(x)\right)^{T} ; \mathscr{D}, B, F(x)-$ те же, что и в задаче (14)-(15).

ЛЕмма 10. Пусть $\lambda$ таково, что $\Delta^{-1}(\lambda)$ существует, $v(x, \lambda)$ - решение (14)-(15), и(x, $\lambda)$ - решение (79)-(80). Тогда

$$
v(x, \lambda)-u(x, \lambda)=-\left(V_{1}(x, \lambda), \ldots, V_{n}(x, \lambda)\right) \Delta^{-1}(\lambda) U(u(x, \lambda)),
$$

əде $U(u)=\left(U_{1}^{T}(u), \ldots, U_{n}^{T}(u)\right)^{T}, U_{i}(u)=P u^{(i-1)}(0, \lambda)+Q u^{(i-1)}(1, \lambda)(i=$ $1, \ldots, n)$.

ДокАЗАТЕльСтво. Вектор-функция $v(x, \lambda)-u(x, \lambda)$ является решением однородной системы $v^{(n)}-\lambda \mathscr{D} v=0$ и поэтому

$$
v(x, \lambda)-u(x, \lambda)=\sum_{j=1}^{n} V_{j}(x, \lambda) c_{j} .
$$

Постоянные векторы $c_{j}$ удовлетворяют системе

$$
\sum_{j=1}^{n} U_{i j}(\lambda) c_{j}=-U_{i}(u(x, \lambda)) \quad(i=1, \ldots, n) .
$$

Находя отсюда $c_{j}$ и подставляя в $(82)$, приходим к (81). Лемма доказана.

Удалим из $S_{\delta_{0}}$ еше точки $\rho_{k}(k=1,2, \ldots)$ вместе с круговыми окрестностями радиуса $\delta_{0}$, для которых $(\alpha+1) \rho_{k}^{n}$ или $(\alpha-1) \rho_{k}^{n}$ являются собственными значениями краевой задачи:

$$
y^{(n)}(x)-\lambda y(x)=0, \quad y^{(i)}(0)=y^{(i)}(1) \quad(i=0, \ldots, n-1),
$$

где $y(x)$ - скалярная функция, и для оставшейся области сохраним обозначение $S_{\delta_{0}}$.

ЛЕмма 11. В области $S_{\delta_{0}}$ справедливы оченки

$$
U_{j}(u(x, \lambda))=\left(O\left(|\rho|^{j-n}\right), O\left(|\rho|^{j-n}\right)\right)^{T}\|f\|_{1} \quad(j=1, \ldots, n) .
$$

Если же в (79) $F(x)=(\chi(x), \chi(1-x))^{T}$, mо

$$
U_{j}(u(x, \lambda))=\left(O\left(|\rho|^{j-n-1}\right), O\left(|\rho|^{j-n-1}\right)\right)^{T} \quad(j=1, \ldots, n) .
$$


ДокАЗАТЕЛЬСТво. Имеем

$$
u_{i}(x, \lambda)=\int_{0}^{1} G\left(x, t, \lambda d_{i}\right)(B F(t))_{i} d t \quad(i=1,2)
$$

где $G(x, t, \lambda)$ - функция Грина краевой задачи $(83),(B F(t))_{i}$ - компоненты векторфункции

$$
B F(t)=\frac{1}{2}((\alpha+1)(f(t)+f(1-t)),(\alpha-1)(f(t)-f(1-t)))^{T} .
$$

Из (86) и известных $[3 ; \S 5]$ оценок

$$
\frac{\partial^{k} G(x, t, \lambda)}{\partial x^{k}}=O\left(\rho^{1-n+k}\right) \quad(k=0, \ldots, n-1)
$$

получаем оценки (84). Если же $f(t)=\chi(t)$, то интегралы в (86) вычисляются и это приводит к оценкам (85). Лемма доказана.

ЛЕмма 12. Для любого $\delta \in(0,1 / 2)$ в области $S_{\delta_{0}}$ справедливы оценки:

$$
\begin{aligned}
& \left\|v_{i}(x, \lambda)-u_{i}(x, \lambda)\right\|_{C[\delta, 1-\delta]}=\|f\|_{1} O\left(|\rho|^{1-n} \sum_{j=1}^{n} \exp \left(-\delta \operatorname{Re} \rho \widetilde{\omega}_{j}\right)\right) \\
& \left\|v_{i}(x, \lambda)-u_{i}(x, \lambda)\right\|_{C[\delta, 1-\delta]}=O\left(|\rho|^{-n} \sum_{j=1}^{n} \exp \left(-\delta \operatorname{Re} \rho \widetilde{\omega}_{j}\right)\right)
\end{aligned}
$$

если $f(x)=\chi(x) \quad(i=1,2)$.

ДокаЗАТЕЛЬСтво. По лемме 10 в силу оценок (84) (см. также лемму 6) имеем

$$
\begin{gathered}
v_{i}(x, \lambda)-u_{i}(x, \lambda)=\sum_{j=1}^{n}\left(\eta_{i, 2 j-1}(x, \lambda) O\left(|\rho|^{j-n}\right)+\eta_{i, 2 j}(x, \lambda) O\left(|\rho|^{j-n}\right)\right)\|f\|_{1} \\
(i=1,2)
\end{gathered}
$$

Из представлений (40) следует, что

$$
\left\|\eta_{i, l}(x, \lambda)\right\|_{C[\delta, 1-\delta]}=O\left(|\rho|^{1-j} \sum_{k=1}^{n} \exp \left(-\delta \operatorname{Re} \rho \widetilde{\omega}_{k}\right)\right) \quad(i=1,2),
$$

где $l=2 j$ или $l=2 j-1(j=1, \ldots, n)$. Из (90) и (91) получаем (88). Оценки (89) получаются аналогично, только вместо (84) надо воспользоваться оценками (85).

ЛЕмма 13. Для любой $f(x) \in L[0,1]$ и любого $\delta \in(0,1 / 2)$ имеет место

$$
\lim _{r \rightarrow \infty}\left\|\Omega_{r}^{0} f\right\|_{C[\delta, 1-\delta]}=0
$$

əде

$$
\Omega_{r}^{0} f=\frac{1}{2 \pi i} \int_{|\lambda|=r} \sum_{i=1}^{2}\left(v_{i}(x, \lambda)-u_{i}(x, \lambda)\right) d \lambda
$$

ur таково, что $\left\{\rho:|\rho|=r^{1 / n}, 0 \leqslant \arg \rho \leqslant 2 \pi / n\right\} \subset S_{\delta_{0}}$. 
ДокАЗАтЕльство. В (93) выполним замену: $\lambda=\rho^{n}, \rho=|\rho| \exp i \varphi, 0 \leqslant \varphi \leqslant$ $2 \pi / n$. Тогда имеем

$$
\begin{aligned}
\Omega_{r}^{0} f & =\frac{n}{2 \pi} \int_{0}^{2 \pi / n} \rho^{n} \sum_{i=1}^{2}\left(v_{i}(x, \lambda)-u_{i}(x, \lambda)\right) d \varphi \\
& =\frac{n}{2 \pi}\left(\int_{0}^{\gamma_{1}}+\int_{\gamma_{1}}^{\gamma_{2}}+\int_{\gamma_{2}}^{\gamma_{3}}+\int_{\gamma_{3}}^{2 \pi / n}\right)
\end{aligned}
$$

Рассмотрим первый интеграл в (94). По лемме 12

$$
\left\|\int_{0}^{\gamma_{1}}\right\|_{C[\delta, 1-\delta]}=\|f\|_{1} O\left(\sum_{j=1}^{n} \int_{0}^{\gamma_{1}}|\rho| \exp \left(-\delta \operatorname{Re} \rho \widetilde{\omega}_{j}\right) d \varphi\right)
$$

Учитывая, что

$$
\begin{aligned}
\int_{0}^{\gamma_{1}} \exp \left(-\delta \operatorname{Re} \rho \widetilde{\omega}_{j}\right) d \varphi & =\int_{0}^{\gamma_{1}} \exp \left(-\delta\left|\rho \widetilde{\omega}_{j}\right| \sin \varphi\right) d \varphi \\
& \leqslant \frac{1}{\delta\left|\rho \widetilde{\omega}_{j}\right|} \int_{0}^{\infty} \exp (-x) d x<\infty
\end{aligned}
$$

заключаем, что правая часть (95) есть $O(1)\|f\|_{1}$. Аналогично оцениваются и остальные интегралы в (94). Тем самым получена оценка:

$$
\left\|\Omega_{r}^{0} f\right\|_{C[\delta, 1-\delta]}=O(1)\|f\|_{1}
$$

Если $f(x)=\chi(x)$, то, воспользовавшись в предыдущих рассуждениях оценками (89) вместо (88), получим

$$
\lim _{r \rightarrow \infty}\left\|\Omega_{r}^{0} \chi\right\|_{C[\delta, 1-\delta]}=0
$$

Из (96) и (97) по теореме Банаха-Штейнгауза вытекает утверждение леммы.

Получим теперь основной результат статьи.

Теорема 4. Пусть ядро оператора $A$ п раз непрерывно дифференцируемо по $x$ и один раз по $t$ при $0 \leqslant t \leqslant x \leqslant 1$, выполняется условие $(2)$ и $\alpha^{2} \neq 1$. Тогда для любой $f(x) \in L[0,1]$ и любого $\delta \in(0,1 / 2)$ имеет место соотношенuе:

$$
\lim _{r \rightarrow \infty} \max _{\delta \leqslant x \leqslant 1-\delta}\left|S_{r}(f, x)-\frac{1}{2} \sigma_{r\left|d_{1}\right|}(f+g, x)-\frac{1}{2} \sigma_{r\left|d_{2}\right|}(f-g, x)\right|=0,
$$

әде $S_{r}(f, x)$ - частичная сумма ряда Фурье функиии $f(x)$ nо с.п.ф. оператора А для тех характеристических чисел $\lambda_{k}$, для которых $\left|\lambda_{k}\right|<r ; \sigma_{r}(f, x)-$ частичная сумма тригонометрического ряда Фурье функиии $f(x)$ для тех номеров $k$, для которых $(2 k \pi)^{n}<r ; g(x)=f(1-x) ; d_{1}=\alpha+1, d_{2}=\alpha-1$; $r$ таково, что $\left\{\rho:|\rho|=r^{1 / n}, 0 \leqslant \arg \rho \leqslant 2 \pi / n\right\} \subset S_{\delta_{0}}$. 
ДокАЗАТЕльство. Так как $R_{\lambda}^{0} f=v_{1}(x, \lambda)+v_{2}(x, \lambda)$ по следствию теоремы 1 , TO

$$
\frac{1}{2 \pi i} \int_{|\lambda|=r} R_{\lambda} f d \lambda-\frac{1}{2 \pi i} \int_{|\lambda|=r}\left(u_{1}(x, \lambda)+u_{2}(x, \lambda)\right) d \lambda=\Omega_{r} f+\Omega_{r}^{0} f .
$$

$$
\begin{aligned}
\text { Но }-\frac{1}{2 \pi i} \int_{|\lambda|=r} R_{\lambda} f d \lambda & =S_{r}(f, x) \text { и в силу }(86),(87) \\
& -\frac{1}{2 \pi i} \int_{|\lambda|=r} u_{1}(x, \lambda) d \lambda=\frac{1}{2} \sigma_{r\left|d_{1}\right|}(f+g, x), \\
& -\frac{1}{2 \pi i} \int_{|\lambda|=r} u_{2}(x, \lambda) d \lambda=\frac{1}{2} \sigma_{r\left|d_{2}\right|}(f-g, x) .
\end{aligned}
$$

Поэтому утверждение теоремы вытекает из (98) и (92) по теореме 3.

СлЕДСТВИЕ 1. Ecли $f(x) \in L[0,1] u f(x)=f(1-x), m o$

$$
\lim _{r \rightarrow \infty} \max _{\delta \leqslant x \leqslant 1-\delta}\left|S_{r}(f, x)-\sigma_{r\left|d_{1}\right|}(f, x)\right|=0 .
$$

$E c \bumpeq u f(x) \in L[0,1] u f(x)=-f(1-x)$, mo

$$
\lim _{r \rightarrow \infty} \max _{\delta \leqslant x \leqslant 1-\delta}\left|S_{r}(f, x)-\sigma_{r\left|d_{2}\right|}(f, x)\right|=0 .
$$

СЛЕДСТВИЕ 2. Если тригонометрический ряд Фурье функции $f(x) \in C[0,1]$ сходится равномерно на замкнутом множестве е $\subset(0,1)$, симметричном относительно $x=1 / 2$ (т.е. если $x \in e$, то и $1-x \in e)$, то ее ряд Фурье по c.n.ф. сходится равномерно нае.

В самом деле, ряд Фурье функции $g(x)=f(1-x)$ сходится равномерно на $e$. Поэтому $\lim _{r \rightarrow \infty} \sigma_{r}(f, x)=f(x)$ и $\lim _{r \rightarrow \infty} \sigma_{r}(g, x)=g(x)$ равномерно на $e$. Поэтому

$$
\frac{1}{2} \sigma_{r\left|d_{1}\right|}(f+g, x)+\frac{1}{2} \sigma_{r\left|d_{2}\right|}(f-g, x)
$$

сходится равномерно на $е$ к $f(x)$ и тогда по теореме 4

$$
\lim _{r \rightarrow \infty} \max _{x \in e}\left|S_{r}(f, x)-f(x)\right|=0 .
$$

\section{Список литературы}

1. Хромов А. П. Теоремы равносходимости для интегро-дифференциальных и интегральных операторов // Матем. сб. 1981. Т. 114 (156). №3. С. 378-405.

2. Хромов А.П. Теорема равносходимости для интегрального оператора с переменным верхним пределом интегрирования // Метрическая теория функций и смежные вопросы анализа. Сборник статей, посвященнњй 70-летию П. Л. Ульянова. М.: Изд-во АФЦ, 1999. C. $255-266$.

3. Наймарк М. А. Линейные диффференциальные операторы. М.: Наука, 1969.

4. Беллман P., Кук К. Дифференциально-разностные уравнения. М.: Мир, 1967.

Саратовский государственный университет им. Н.Г. Черньшшевского

E-mail: KhromovAP@info.sgu.ru 29.01.2001 